\title{
Evaluation of maxillary arch dimensions and palatal morphology in mouth-breathing children by using digital dental casts
}

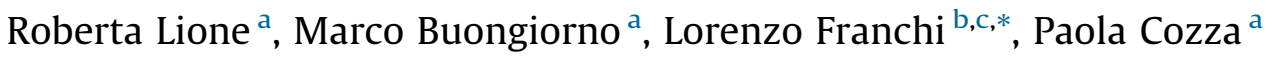 \\ a Department of Orthodontics, University of Rome "Tor Vergata”, Rome, Italy \\ ${ }^{\mathrm{b}}$ Department of Surgery and Translational Medicine, Orthodontics, University of Florence, Florence, Italy \\ ${ }^{\mathrm{c}}$ Thomas M. Graber Visiting Scholar, Department of Orthodontics and Pediatric Dentistry, School of Dentistry, The University of Michigan, Ann Arbor, \\ United States
}

\section{A R T I C L E I N F O}

\section{Article history:}

Received 6 August 2013

Received in revised form 14 September 2013

Accepted 16 September 2013

Available online $\mathrm{xxx}$

\section{Keywords:}

Palatal morphology

Mouth-breathing pattern

Digital dental casts

\begin{abstract}
A B S T R A C T
Objective: The aim of the present study was to analyze the variations of maxillary arch size and of palatal morphology in subjects with prolonged mouth-breathing due to allergic rhinitis when compared with a control group with normal breathing pattern by using a three-dimensional analysis on digital casts. Methods: 26 Caucasian children (19 females and 7 males) with a mean age of 8.5 years (SD 1.6 years) were selected according to the following criteria: mouth-breathing pattern due to allergic rhinitis, early mixed dentition, skeletal Class I relationship and prepubertal stage of cervical vertebral maturation. The study group was compared with a control group of 17 nasal breathing subjects ( 9 females; 8 males, mean age 8.5 years SD 1.7 years). For each subject an initial dental cast was taken and the upper arch was scanned by using a tridimensional scanner. On each digital model linear measurements were performed to analyze maxillary arch dimensions and palatal morphology. Significant between-group differences were tested with the Student $t$-test $(p<0.05)$.

Results: the transverse dimension of the upper arch was significantly smaller in subjects of the study group thus confirming the influence of oral breathing on skeletal development with a significant constriction of the whole palate. The study group showed a higher and sharper palatal vault at the level of second deciduous molars and of first permanent molars.

Conclusions: Children with mouth-breathing pattern showed a significant constriction of the maxillary arch and an increased palatal height when compared with subjects with normal breathing pattern.
\end{abstract}

(c) 2013 Elsevier Ireland Ltd. All rights reserved.

\section{Introduction}

The development of the craniofacial structures is the result of the continuous interactions between genetic and environmental factors. Mouth-breathing is a pathological alteration of the normal breathing pattern [1]. Prolonged mouth-breathing can produce muscular and postural alterations, which interacting with the craniofacial structures, can cause alterations on the morphology, position, and growth direction of the jaws [2]. Mouth-breathing causes the tongue to rest in a low position in the oral cavity. This will result in an imbalance of forces between the cheeks and the tongue, which can directly affect the growth and development of the upper and the lower jaws [3]. In mouth-breathing patients the tongue does not exert any force on the upper teeth, which allows

\footnotetext{
* Corresponding author at: Department of Surgery and Translational Medicine, Orthodontics, Università degli Studi di Firenze, Via del Ponte di Mezzo, 46-48, Firenze 50127, Italy. Tel.: +39 3333602195.

E-mail address: lorenzo.franchi@unifi.it (L. Franchi).
}

the upper arch to remain undeveloped, directly influencing the skeletal development in preschool children [4]. Although mouthbreathing etiology is multifactorial, the most common causes are anatomical obstruction, such as palatine and pharyngeal tonsil hypertrophy, allergic rhinitis, nasal septal deviation, nasal polyps and nasal turbinate hypertrophy [5]. In particular allergic rhinitis, a chronic respiratory problem with a high prevalence, result from a complex, allergen-driven mucosal inflammatory process that can result in nasal obstruction with consequent transition from nasal to oral breathing [6].

In the literature the effects of the alteration of the normal breathing pattern on dental arches have generally been described as dimensional changes in intercanine and intermolar width and arch lengths by using traditional methods on dental casts measured with calipers [3-5,7,8]. Vieira et al. [3] and Harari et al. [6] found that a change in the breathing pattern of children can induce dental changes such as a narrowing of the maxillary arch at the level of the canine. Bresolin et al. [7] as well Mattar et al. [8], demonstrated that mouth-breathing individuals showed greater palatal height and narrower intermolar width than did

0165-5876/\$ - see front matter (c) 2013 Elsevier Ireland Ltd. All rights reserved. http://dx.doi.org/10.1016/j.ijporl.2013.09.028 
nasal-breathing subjects. However, changes measured with a traditional measuring approaches might not always highlight the modification in the arch form $[9,10]$ and these methods could not exclude bias in assessing the transverse dimension of the maxillary arch due to improper tooth position, such as the buccal tooth tipping $[11,12]$. To overcome these problems, an optical scanner can be used to obtain three-dimensional images of dental casts [13]. In fact, recent advances in digital technology have vastly improved the diagnostic phase of orthodontic treatment, and analog records have quickly been replaced by digital formats [14]. Several studies in the literature have verified the accuracy of angular and linear measurements on three-dimensional digital models with different software [15,16]. Three-dimensional images allow to assess linear and angular measurements that describe the arch form and the morphology of the palatal vault [16].

The aim of the present study was to analyze the variations of maxillary arch dimensions and of palatal morphology in a group of subjects with prolonged mouth-breathing due to allergic rhinitis when compared with a control group of subjects with normal breathing pattern and occlusion by using a three-dimensional analysis on digital dental casts.

\section{Materials and methods}

26 Caucasian children ( 19 females; 7 males) with a mean age of 8.5 years (SD 1.6 years) who sought orthodontic treatment at the Department of Orthodontics at the University of Rome "Tor Vergata" were selected. The inclusion criteria for the enrollment of the subjects in the study group (SG) were mouth-breathing pattern due to allergic rhinitis, early mixed dentition with either a Class I or end-to-end molar relationship, skeletal Class I relationships and prepubertal stage of cervical vertebral maturation as assessed by the cervical vertebral maturation method (CS1, CS2) [17]. Mode of breathing was defined by an otorhinolaryngologist according to complete physical examination including skin testing, anterior rhinoscopy, rhinomanometry to measure nasal airflow and pressure during respiration, flexible nasopharyngoscopy or nasopharyngeal X-ray, and to history confirmed by questionnaire answered by the patients' parents. Only at the end of the complete examination the otorhinolaryngologist classified subjects as showing normal respiratory pattern or as exclusive mouth breathers. $44 \%$ of the subjects of the study group presented with palatine and/or pharyngeal tonsil hypertrophy. However, the pathological characteristic that was found in all subjects of the study sample was prolonged allergic rhinitis. Probably as a consequence of mode-breathing, 13 subjects of the study group presented with a unilateral posterior cross-bite, 8 with a bilateral posterior cross-bite and 5 without posterior cross-bite. In 5 patients with unilateral posterior crossbite an associated openbite due to lack of space for the complete eruption of the upper incisors was observed.

Exclusion criteria were: sucking habits, previous history of nasal respiratory surgery, previous orthodontic treatment, lippalate cleft, and other genetic diseases. This project was approved by the Ethical Committee at the University of Rome, "Tor Vergata", and informed consent was obtained from the patients' parents.

The study group was compared with a control group of 17 prepubertal subjects ( 9 females; 8 males) presenting with normal breathing pattern and mean age of 8.5 years (SD 1.7 years). The control group matched the study group as to occlusal development, skeletal and occlusal relationships, and skeletal maturation. No subjects of the control group showed either transverse or vertical skeletal discrepancies.

For each subject of the study and control groups initial dental casts were available. In order to analyze the maxillary arch form and the palatal morphology, the upper dental casts were scanned

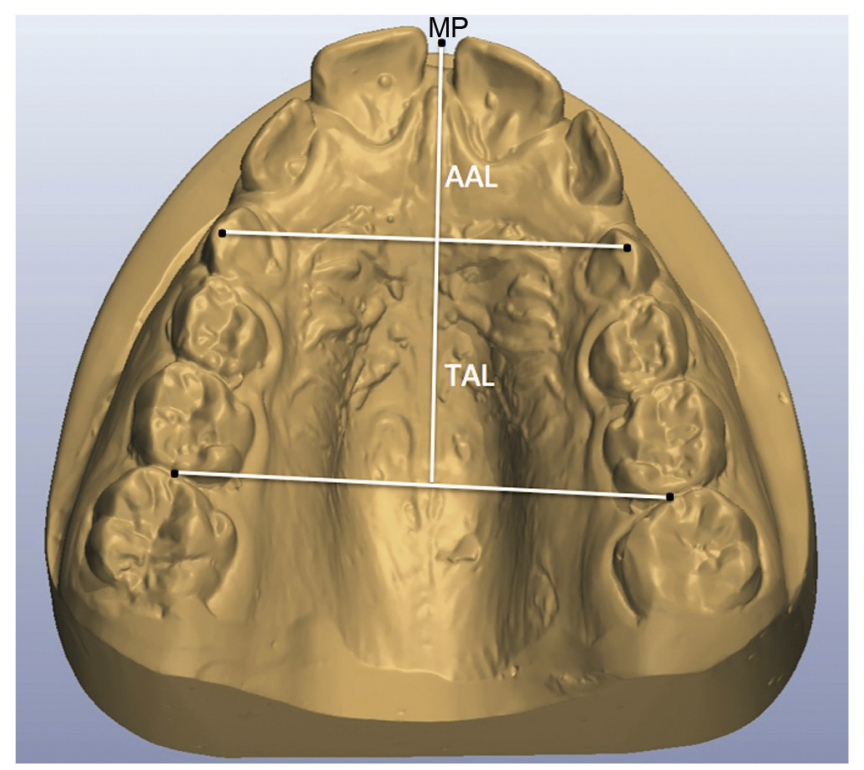

Fig. 1. Conventional measurements: AAL: distance from the midpoint (MP) to a line joining the cusps of upper deciduous canines; TAL: distance from MP to a line joining the mesial surfaces of the first permanent molars.

by a tridimensional scanner (D800, 3Shape A/S, Copenhagen $\mathrm{K}$ Denmark, Scan time $25 \mathrm{~s}$, Resolution 2 cameras 5.0 megapixels, Ultra high point accuracy $<15$ microns). Each cast was scanned from 10 or more views that were then combined and rendered into three-dimension by using a specific software (3shape-ScanItOrthodontics ${ }^{\mathrm{TM}} 2010$-2p3, 3Shape A/S, Copenhagen K, Denmark). The virtual three-dimensional models were measured and analyzed with a specific software (3Shape-OrthoAnalyzer ${ }^{\mathrm{TM}} 2010$, 3Shape A/S, Copenhagen K, Denmark).

To analyze the transverse and the sagittal dimensions of the maxillary arch, a midpoint (MP) was identified on each digital model as the midpoint between the most mesial point of the incisal margin of both central incisors. Subsequently, conventional linear measurements according to Bu et al. [18], and Ahn et al. [11] were performed (Fig. 1):

\section{- Anterior Arch Length (AAL): distance from MP to a line joining the cusp tips of the deciduous canines; \\ - Total Arch Length (TAL): distance from MP to a line joining the midpoints of the mesial margins of the first permanent molars.}

As previously described by Primozic et al. (2013) [12,13], a gingival plane was constructed by connecting the center of the dento-gingival junction of all teeth (Fig. 2). After the identification of the gingival plane the following measurements were performed:

- D3: distance between the centers of dento-gingival junctions of right and left deciduous canines;

- D4: distance between the centers of dento-gingival junctions of right and left first deciduous molars;

- D5: distance between the centers of dento-gingival junctions of right and left second deciduous molars;

- D6: distance between the centers of dento-gingival junctions of right and left first permanent molars;

- H3: distance between the gingival plane and the highest point of the palatal vault on the midpalatal rafe at the level of deciduous canines;

- H4: distance between the gingival plane and the highest point of the palatal vault on the midpalatal at the level of first deciduous molars; 


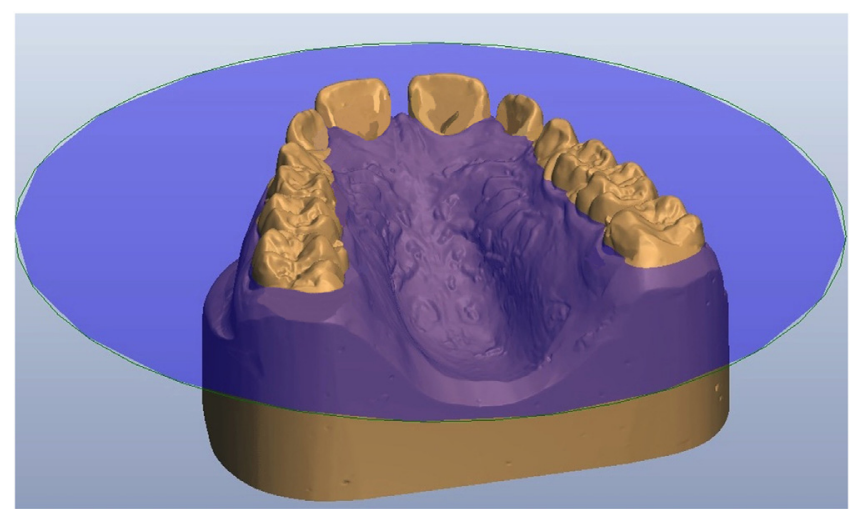

Fig. 2. Gingival plane constructed by connecting the midpoints of the dentogingival junction of all teeth.

- H5: distance between the gingival plane and the highest point of the palatal vault on the midpalatal rafe at the level of second deciduous molars;

- H6: distance between the gingival plane and the highest point of the palatal vault on the midpalatal rafe at the level of first permanent molars;

- A3: angle between the lines connecting the centers of the dentogingival junctions and the highest point of the palatal vault on the midpalatal rafe at the level of right and left deciduous canines;

- A4: angle between the lines connecting the centers of the dentogingival junctions and the highest point of the palatal vault on the midpalatal rafe at the level of right and left first deciduous molars;

- A5: angle between the lines connecting centers of the dentogingival junctions and the highest point of the palatal vault on the midpalatal rafe at the level of right and left second deciduous molars;

- A6: angle between the lines connecting centers of the dentogingival junctions and the highest point of the palatal vault on the midpalatal rafe at the level of right and left first permanent molars (Fig. 3).

\section{Statistical analysis}

To determine the reliability of the method, measurements on the digital models were performed by one trained examiner (M.B.) and repeated after an interval of approximately two weeks. A paired $t$-test was used to compare the two measurements (systematic error). The magnitude of the random error was calculated by using the method of moments' estimator [19].

The power of the study for the independent sample $t$ test was calculated on the basis of sample size of 2 groups and an effect size [20] equal to 0.9. The power was 0.80 at an alpha level of 0.05 (SigmaStat 3.5, Systat Software, Point Richmond, CA).

Descriptive statistics were calculated for all the measurements in each group. Exploratory statistics revealed that all variables were normally distributed (Kolmogorov-Smirnov test) with equality of variances (Levene's test). Significant between-group differences were tested with the Student $t$-test. Statistical significance was set at $P<0.05$ (Table 1). All statistical computations were performed by using a specific software (SigmaStat 3.5, Systat software, Point Richmond, CA).

\section{Results}

No systematic error was found between the repeated measurements. The $p$ value for the paired $t$ test ranged from $0.064(\mathrm{H} 3)$ to

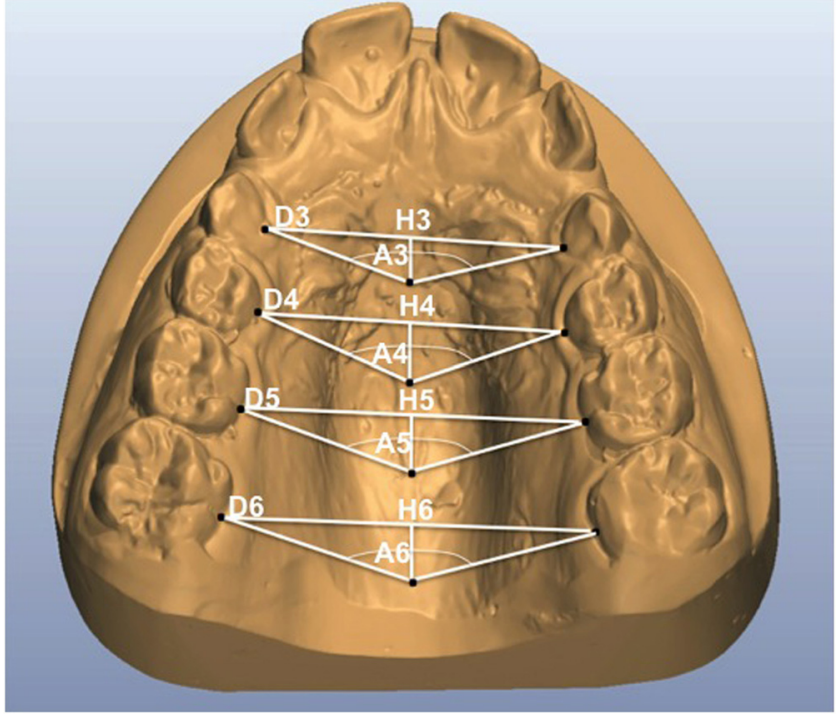

Fig. 3. Analysis of the palatal surface: D3: distance between the centers of dentogingival junctions of right and left deciduous canines; D4: distance between the centers of dento-gingival junctions of right and left first deciduous molars; D5: distance between the centers of dento-gingival junctions of right and left second deciduous molars; D6: distance between the centers of dento-gingival junctions of right and left first upper molars; H3: distance between the gingival plane and the highest point of the palatal vault on the midpalatal rafe at the level of upper deciduous canines; H4: distance between the gingival plane and the highest point of the palatal vault on the midpalatal at the level of first upper deciduous molars; $\mathrm{H} 5$ : distance between the gingival plane and the highest point of the palatal vault on the midpalatal rafe at the level of second upper deciduous molars; H6: distance between the gingival plane and the highest point of the palatal vault on the midpalatal rafe at the level of first upper molars; A3: angle between the centers of the dento-gingival junctions and the highest point of the palatal vault on the midpalatal rafe at the level of right and left deciduous canines; A4: angle between the centers of the dento-gingival junctions and the highest point of the palatal vault on the midpalatal rafe at the level of right and left first upper deciduous molars; A5: angle between the centers of the dento-gingival junctions and the highest point of the palatal vault on the midpalatal rafe at the level of right and left second upper deciduous molars; A6: angle between the centers of the dento-gingival junctions and the highest point of the palatal vault on the midpalatal rafe at the level of right and left first upper molars.
Table 1

Descriptive statistics and between-group differences for all measurements.

\begin{tabular}{|c|c|c|c|c|c|}
\hline \multirow[t]{2}{*}{ Variables } & \multicolumn{2}{|c|}{$\begin{array}{l}\text { SG (study group) } \\
N=26\end{array}$} & \multicolumn{2}{|c|}{$\begin{array}{l}\text { CG (control } \\
\text { group) } N=17\end{array}$} & \multirow{2}{*}{$\begin{array}{l}\text { Significance } \\
p\end{array}$} \\
\hline & Mean & SD & Mean & SD & \\
\hline$A A L \mathrm{~mm}$ & 9.0 & 2.7 & 9.1 & 1.2 & ns \\
\hline TAL mm & 27.9 & 2.8 & 28.7 & 1.3 & ns \\
\hline D3 mm & 21.7 & 2.1 & 25.8 & 1.6 & ${ }^{* *}$ \\
\hline $\mathrm{D} 4 \mathrm{~mm}$ & 22.8 & 2.4 & 27.8 & 1.9 & ${ }^{* *}$ \\
\hline D5 $\mathrm{mm}$ & 25.4 & 3.0 & 31.8 & 2.3 & $* *$ \\
\hline D6 $\mathrm{mm}$ & 29.0 & 3.0 & 35.5 & 2.6 & ${ }^{* *}$ \\
\hline $\mathrm{H} 3 \mathrm{~mm}$ & 4.4 & 1.9 & 5.0 & 1.5 & ns \\
\hline $\mathrm{H} 4 \mathrm{~mm}$ & 10.6 & 1.8 & 10.5 & 1.5 & ns \\
\hline $\mathrm{H} 5 \mathrm{~mm}$ & 13.8 & 1.6 & 12.8 & 1.3 & * \\
\hline $\mathrm{H} 6 \mathrm{~mm}$ & 13.4 & 2.6 & 11.8 & 1.4 & * \\
\hline A3 deg & 136.8 & 16.0 & 138.3 & 11.5 & ns \\
\hline A4 deg & 94.5 & 8.8 & 106.2 & 9.1 & ${ }^{* *}$ \\
\hline A5 deg & 86.3 & 8.4 & 102.2 & 7.1 & $* *$ \\
\hline A6 deg & 95.0 & 13.2 & 112.7 & 6.5 & ${ }^{* *}$ \\
\hline
\end{tabular}

ns: not significant.

${ }_{* *}^{*} p<0.05$.

$p<0.001$ 
0.855 (D6). The random error for the linear measurements ranged from $0.09 \mathrm{~mm}(\mathrm{H} 3)$ to $0.28 \mathrm{~mm}$ (D4) and for the angular measurements ranged from $0.30^{\circ}$ (A3) to $0.41^{\circ}$ (A5). Descriptive statistics and significant between-group differences are given in Table 1.

No statistical significant differences in the sagittal measurements were found between the two groups (AAL: $9.0 \mathrm{~mm}$ in SG vs $9.1 \mathrm{~mm}$ in CG; TAL: $27.9 \mathrm{~mm}$ in SG vs $28.7 \mathrm{~mm}$ in CG) while the transverse dimension of the upper arch was significantly smaller in SG vs CG (D3: $21.7 \mathrm{~mm}$ in SG vs $25.8 \mathrm{~mm}$ in CG; D4: $22.8 \mathrm{~mm}$ in SG vs $27.8 \mathrm{~mm}$ in CG; D5: $25.4 \mathrm{~mm}$ in SG vs $31.8 \mathrm{~mm}$ in CG; D6, $29.0 \mathrm{~mm}$ in SG vs $35.5 \mathrm{~mm}$ in $\mathrm{CG}$ ).

The height of the palatal vault was significantly greater in SG vs CG at the level of second deciduous molars and of first permanent molars (H5: $13.8 \mathrm{~mm}$ in SG vs $12.8 \mathrm{~mm}$ in CG; H6: $13.4 \mathrm{~mm}$ in SG vs $11.8 \mathrm{~mm}$ in CG) while no differences were found at the level of deciduous canines and first deciduous molars (H3: $4.4 \mathrm{~mm}$ in SG vs $5.0 \mathrm{~mm}$ in CG; H4: $10.6 \mathrm{~mm}$ in SG vs $10.5 \mathrm{~mm}$ in CG).

The study group showed significantly smaller angular measurements with respect to CG (A4: $94.5^{\circ}$ in SG vs 106.2 in CG; A5: $86.3^{\circ}$ in $\mathrm{SG}$ vs $102.2^{\circ}$ in CG; A6: $95.0^{\circ}$ in SG vs $112.7^{\circ}$ in CG), with the exception of the angular value in the most anterior part of the palate at the level of the deciduous canines (A3: $136.8^{\circ}$ in SG vs $138.3^{\circ}$ in CG) that exhibited no significant difference between the 2 groups.

\section{Discussion}

The aim of the present study was to analyze the variations of the maxillary arch and palatal vault in a group of subjects with a mouth-breathing pattern due to allergic rhinitis (study group, SG) when compared with a control group of subjects with normal breathing pattern (control group, CG). Although numerous studies have investigated the skeletal and occlusal characteristics of mouth-breathing individuals [2-4,6-8], no study evaluated the morphology of the palatal vault in mouth- versus nasal-breathing subjects by means of digital 3D dental casts. 3D images can be used to assess linear and angular measurements that describe the arch form and the palatal morphology [21].

In the present study no differences were found between SG and CG in terms of sagittal arch length, suggesting that prolonged abnormal breathing pattern does not affect the sagittal dimension of the maxillary arch. However, with reference to the transverse dimensions of the maxillary arch, it was observed that the intermolar and intercanine distance was significantly smaller in mouth-breathing patients when compared with that of nasal breathers. This finding indicated a greater narrowing of the maxillary arch especially in the posterior part of the palate in SG. These results were similar to those obtained by Harari et al. [5], Bresolin et al. [7] and Berwig at al. [22] who compared sample groups of nasal and mouth breathers in the 8-12 year age on plaster casts demonstrating that a change in the breathing pattern of children leads to a narrowing of intermolar and intercanine widths. The current study showed that in mouth-breathing subjects also the transverse widths measured on the gingival plane were smaller when compared with the control group. As reported by Primozic et al. [12,13], measurements on digital dental casts at the level of the gingival plane allow a more accurate analysis of the transverse dimension of maxillary arch because this method of evaluation exclude bias due to improper teeth position as buccal tipping. In the present study a constriction of the maxillary arch was observed with an increasing gradient from the posterior part of the palate at the level of the first upper molars to the anterior part of the palate at the level of the upper deciduous canines (D3 -4.1 $\mathrm{mm}$ in SG vs CG; D4 $-5.0 \mathrm{~mm}$ in SG vs CG; D5 $-6.4 \mathrm{~mm}$ in SG vs CG; D6 -6.5 mm in SG vs CG). In contrast Vieira et al. [3] analyzing 29 mouth-breathing children and 15 nasalbreathing subjects with a mean age of 5 years on plaster casts, reported similar transverse dimensions between nasal-breathing and mouth-breathing groups.

As for the palatal height, mouth-breathing subjects showed significant increases only in the posterior part of the palate at the level of first permanent molars and at the level of second deciduous molars (H5: $+1.0 \mathrm{~mm}$ in SG vs CG; H6: $+1.6 \mathrm{~mm}$ in SG vs CG). These results are in agreement with studies involving children with and without allergic rhinitis of Freitas et al. [23] and Ghasempour et al. [24] who observed that the palatal height was significantly greater in children with allergic rhinitis than in nasal-breathing group. In contrast Vieira et al. [3] investigated white preschool individuals and found that mouth-breathing had no impact on palatal height. These differences can be attributed to different methods of evaluations on digital and plaster casts model and to different severity of nasal airway obstruction, as in the present study only exclusive mouth-breathers were included in the study group.

Finally, digital plaster casts allowed to analyze the morphology of the palate by measuring the angles between the highest point of the palatal vault on the midpalatal rafe and the centers of the dento-gingival junctions. These angles were significantly smaller in mouth-breathers in the whole palate (with the exception of the angle at the level of deciduous canines) thus demonstrating a higher and sharper palatal morphology associated with a prolonged abnormal breathing pattern.

\section{Conclusion}

- Children with mouth-breathing pattern showed a significant constriction of the maxillary arch with an increasing gradient from the anterior to the posterior part of the palate when compared with a control group of subjects with normal breathing pattern;

- The palatal height was significantly increased in mouthbreathing subjects in the posterior region of the palate;

- The palatal vault in subjects with mouth-breathing pattern showed a higher and sharper morphology especially at the level of the first permanent molars.

\section{References}

[1] F.P. Van Der Linden, Genetic and environmental factors in dentofacial morphology, Am. J. Orthod. 52 (1966) 576-583.

[2] M. Baroni, F. Ballanti, L. Franchi, P. Cozza, Craniofacial features of subjects with adenoid, tonsillar, or adenotonsillar hypertrophy, Prog. Orthod. 12 (2011) 38-44.

[3] B.B. Vieira, A.C.M. Sanguino, S.E. Mattar, C.E. Itikawa, W.T. Anselmo-Lima, F.C.P. Valera, et al., Influence of adenotonsillectomy on hard palate dimensions, Int. J. Pediatr. Otorhinolaryngol. 76 (2012) 1140-1144.

[4] T. Peltomaki, The effect of mode of breathing on craniofacial growth-revisited, Eur. J. Orthod. 29 (2007) 426-429.

[5] D. Harari, M. Redlich, S. Miri, T. Hamud, M. Gross, The effect of mouth breathing versus nasal breathing on dentofacial and craniofacial development in orthodontic patients, Laryngoscope 120 (2010) 2089-2093.

[6] R.A. Settipane, C. Schwindt, Chapter 15: Allergic rhinitis, Am. J. Rhinol. Allergy 27 (Suppl. 1) (2013) 52-55.

[7] D. Bresolin, P.A. Shapiro, G.G. Shapiro, M.K. Chapko, S. Dassel, Mouth breathing in allergic children: its relationship to dentofacial development, Am. J. Orthod. 83 (1983) 334-340

[8] S.E. Mattar, W.T. Anselmo-Lima, F.C. Valera, M.A. Matsumoto, Skeletal and occlusal characteristics in mouth-breathing pre-school children, J. Clin. Pediatr. Dent. 28 (2004) 315-318.

[9] J. Henrikson, M. Persson, B. Thilander, Long-term stability of dental arch form in normal occlusion from 13 to 31 years of age, Eur. J. Orthod. 23 (2001) 51-61.

[10] S. Petren, L. Bondemark, Correction of unilateral posterior crossbite in the mixed dentition: a randomized controlled trial, Am. J. Orthod. 133 (2008) 790e7$790 \mathrm{e} 13$.

[11] J.S. Ahn, M.S. Park, H.C. Song, Y.S. Park, Three-dimensional interpretation of intercanine width change in children: a 9-year longitudinal study, Am. J. Orthod. 142 (2012) 323-332.

[12] J. Primozic, S. Richmond, C.H. Kau, A. Zhurov, M. Ovsenik, Three-dimensional evaluation of early crossbite correction: a longitudinal study, Eur. J. Orthod. 35 (2013) 7-13. 
[13] J. Primozic, T. Baccetti, L. Franchi, S. Richmond, F. Farnik, M. Osvenik, Threedimensional assessment of palatal change in a controlled study of unilateral posterior crossbite correction in the primary dentition, Eur. J. Orthod. 35 (2013) 199-204.

[14] K.G. Lightheart, J.D. English, C.H. Kau, S. Akyalcin, H.J. Bussa, K.R. McGrory, et al., Surface analysis of study models generated from Ortho CAD and cone-beam computed tomography imaging, Am. J. Orthod. 141 (2012) 686-693.

[15] M.V. Sousa, E.C. Vasconcelos, G. Janson, D. Garib, A. Pinzan, Accuracy and reproducibility of 3-dimensional digital model measurements, Am. J. Orthod. 142 (2012) 269-273.

[16] A.P. Sjögren, J.E. Lindgren, J.A. Huggare, Orthodontic study cast analysis-reproducibility of recordings and agreement between conventional and 3D virtual measurements, J. Digit. Imaging 23 (2010) 482-492.

[17] T. Baccetti, L. Franchi, J.A. McNamara, The cervical vertebral maturation (CMV) method for the assessment of optimal treatment timing in dentofacial orthopedics, Semin. Orthod. 11 (2005) 119-129.
[18] X. Bu, K. Khalaf, R.S. Hobson, Dental arch dimensions in oligodontia patients, Am. Orthod. 134 (2008) 768-772.

[19] S.D. Springate, The effect of sample size and bias on the reliability of estimates of error: a comparative study of Dahlberg's formula, Am. J. Orthod. 83 (1983) 382-390.

[20] J. Cohen, A power primer, Psychol. Bull. 112 (1992) 155-159.

[21] O.Zilberman, J.A.V. Huggare, K.A. Parakakis, Evaluation of the validity of tooth size measurements using conventional and three dimensional virtual orthodontic models, Angle Orthod. 73 (2003) 301-306.

[22] L.C. Berwig A.M. Silva, E.C. Correa, A.B. Moraes, M.M. Montenegro, R.A. Ritzel, Hard palate dimensions in nasal and mouth breathers from different etiologies, J. Soc. Bras. Fonoaudiol. 23 (2011) 308-314.

[23] F.C.N. Freitas, E.P. Bastos, L.S. Primo, V.L. Freitas, Evaluation of the palate dimensions of patients with perennial allergic rhinitis, Int. J. Paediatr. Dent. 11 (2001) 365-371.

[24] M. Ghasempour, S. Mohammadzadeh, S. Garakani, Palatal arch diameters of patients with allergic rhinitis, Iran. Allergy Asthma Immunol. 8 (2009) 63-64. 